\title{
Uncertainties in Ultrasonic Particle Sizing in Solid-in-Liquid Suspensions
}

Raied S. Al-Lashi* and Richard E. Challis**

* Electronics and Computer Science (ECS) Department, Faculty of Physical Sciences and Engineering, University of Southampton,UK

**Electrical Systems and Optics Division, Faculty of Engineering, University of Nottingham, UK.

\section{Correspondence Email: $\underline{\text { R.Al-Lashi@soton.ac.uk }}$}

\begin{abstract}
Measurements of the frequency dependence of ultrasonic attenuation can be used as the basis for the estimation of particle size distributions (PSDs) in solid-in-liquid suspensions. The method requires matching the attenuation simulated by a candidate PSD in combination with a wave propagation model to the measured function in a fitting procedure. Uncertainty in the type of candidate PSD, whether based on fractional volume or fractional number of the dispersed particles, can cause errors in the overall estimation process, particularly for the median particle size. These uncertainties are investigated in the first part of this paper. The second part deals with uncertainties associated with the values for the physical properties of the suspended particles, seven of which are required in the simulation stage. It is shown that the particle sizing exercise is relatively insensitive to all of the physical properties except density, for which values are necessary to an accuracy commensurable with that required for the two principal parameters associated with the PSD - median size and standard deviation. The discussion is limited to small (less than 1 micron) silica particles dispersed in water. The results will have more general application.
\end{abstract}

\section{INTRODUCTION}

Colloidal mixtures consist of small particles dispersed in a surrounding liquid with particle sizes in the range $10 \mathrm{~nm}$ to $100 \mu \mathrm{m}$ [1]. If the particles are solid the mixture is known as a suspension or slurry, and if they are in the form of liquid droplets the mixture is known as an emulsion. A monodisperse mixture contains particles of a single size, and a polydisperse mixture contains a range of particle sizes which is characterised by a particle size distribution (PSD). Many commercial materials either exist in colloidal form or pass through a colloidal stage during their manufacture. The PSD is an important measure of product quality because it determines such things as stability and shelf life, as well as the ultimate functionality of the material. There are frequent requirements to measure PSD for the purposes of process 
control, quality assurance testing, or basic laboratory investigation. Ultrasonic measurements of attenuation or phase velocity as functions of frequency can be used to estimate PSD. They have the advantage over optical techniques that they can be applied to mixtures that are optically opaque [2]. The technique has been used by, for example, Davis [3] to measure mass flow and particle size in coal slurries, McClements and Povey [4] to examine aqueous sunflower oil emulsions in the context of the food industry, and Holmes et al [5] to study aqueous suspensions of polystyrene and silica. It is generally recognised that ultrasonic wave attenuation is more sensitive than phase velocity to dispersed particle size [4], and so attenuation is the preferred variable for particle sizing [6]. There exists an international standard which applies to particle characterisation by means of ultrasound attenuation spectroscopy [7].

In the ultrasonic method, the attenuation coefficient is measured as a function of frequency, typically between $1 \mathrm{MHz}$ and a few 10s of $\mathrm{MHz}$. A mathematical model is run to simulate the measured attenuation function, see [8]; it has as its inputs the physical properties of the continuous and disperse phases in the mixture and a candidate PSD function in terms of either the fractional volume occupied in the mixture by particles in given size ranges, or the number of particles in given size ranges - the so-called volume and number distributions. The model is adapted by changing the parameters of the candidate PSD systematically until the best match is obtained between the measured and simulated attenuation functions of frequency. The match is obtained in a least-squared-error sense [7], typically using the Marquardt algorithm $[9,10]$. At this point the adapted candidate PSD is taken to represent the real PSD in the test mixture.

There are many potential sources of error in this method: (i) The measured attenuation is relatively featureless as a function of frequency and may be prone to bias and variance (noise) errors, see Kalashnikov and Challis [11]. (ii) The underpinning mathematical model is typically based on Epstein and Carhart [12] and Allegra and Hawley [13] , the so-called ECAH model. Its implementation can take many forms with various degrees of approximation which can affect the fidelity of computed results. (iii) There may be uncertainty or misunderstanding of the choice between volume and number based size distributions. (iv) The modelling stage typically requires seven physical parameters to describe each phase in the test mixture. 
For many materials these are available from standard reference works [14, 15], but there remain many materials of industrial significance where the required properties are unknown, immeasurable, or at best extremely difficult and expensive to obtain. Babick et al investigated two strategies to measure PSD for emulsions with partly unknown properties [16]. The first was based on fitting modelled sound speed as well as attenuation to the measured data. The second strategy was based on a statistical approach to determine an empirical model. They found that the applicability of these approaches depended on the nature of the disperse phase, the range of particle sizes compared to the ultrasonic wavelengths, and the particle concentration. It may be necessary to make estimates (intelligent guesses) of the unknown properties, and this process implies uncertainty and potential error.

\section{TYPES OF PARTICLE SIZE DISTRIBUTIONS}

Ideally, the form of the candidate PSD should match the form of the physical PSD in the test mixture, and here a number of functions have been suggested to describe appropriate PSDs [17]. Some of these have been derived from pragmatic examination of the closeness of fit with measured particle size frequency distributions, and others have been derived from distribution functions of a general statistical nature. Among the latter are the Gaussian (normal) and lognormal distributions. Many measurements produce a more or less skewed distribution especially when mean values are low and variances are large [18]. Many real suspensions and emulsions can be described simply when expressed in terms of the logarithm of the particle radius, rather than on a linear radius scale. This is an empirical observation, which probably results from the physical and chemical nature of the production of the suspension or emulsion, see Kiss et al [19]. The use of the log-normal distribution in particle system characterisation is detailed in a key international standard [20]. The basic ideas behind the distribution follow:

The log-normal distribution function $q_{r}(x)$ in particle size expresses the probability that a particle will occupy a size range $x$ to $x+d x$ [20]

$$
q_{r}(x)=\frac{1}{x \sigma \sqrt{2 \pi}} \exp \left[-\frac{1}{2 \sigma^{2}}\left[\ln x-\ln x_{50, r}\right]^{2}\right]
$$

where $x$ is the particle radius, $r$ is the dimensionality (type of quantity) of a distribution, $r=0$ : number, $r=1$ : length, $r=2$ : area, $r=3$ : volume or mass, $x_{50, r}$ is the median particle size of a distribution of dimensionality $r$ and $\sigma$ is the dimensionless standard deviation of $\ln (x)$. The 
complete $k$ th moment $M_{k, r}$ of a log-normal distribution of a dimensionality $r$ can be defined as[20]

$$
M_{k, r}=x_{50, r}^{k} e^{0.5 k^{2} \sigma^{2}}=e^{k \ln x_{50, r}+0.5 k^{2} \sigma^{2}}
$$

For a given particulate mixture, there are transformations which relate a distribution of dimension $p$ to one of dimension $r$ [21], as follows

$$
q_{r}(x)=\frac{x^{r-p} q_{p}(x)}{M_{r-p, p}}
$$

where $r$ and $p$ are either $0,1,2$ or 3. This conversion results in a change in the overall shape of the distribution due to the change in the height of the density distribution at a certain size $x$ [21]. For instance, a volume distribution $(r=3)$ can be obtained from a number distribution $(p=0)$ by

$$
q_{3}(x)=\frac{x^{3} q_{0}(x)}{e^{3 \ln x_{50,3}+4.5 \sigma^{2}}}
$$

The resulting volume density distribution is shifted towards the larger particles. This shift is proportional to the distribution width [21]. The general relationship for particle median sizes of different dimensionalities can be expressed as

$$
x_{50, r}=x_{50, p} e^{(r-p) \sigma^{2}}
$$

On logarithmic abscissa, eq. (5) becomes

$$
\ln x_{50, r}=\ln x_{50, p}+(r-p) \sigma^{2}
$$

The log-normal distributions of different dimensionalities, $r$, are related by [20]

$$
q_{r}(x)=\frac{1}{x \sigma \sqrt{2 \pi}} \exp \left[-\frac{1}{2 \sigma^{2}}\left[\ln x-\left(\ln x_{50, p}+(r-p) \sigma^{2}\right)\right]^{2}\right]
$$

Thus the log-normal distributions shifts by $(r-p) \sigma^{2}$ depending on the direction of the transformation, and their widths remain unchanged. For instance, if $r=3$ and $p=0$, the lognormal volume distribution $q_{3}(x)$ is obtained from the log-normal number distribution $q_{0}(x)$ by shifting the latter towards larger sizes by $+3 \sigma^{2}$

$$
q_{3}(x)=\frac{1}{x \sigma \sqrt{2 \pi}} \exp \left[-\frac{1}{2 \sigma^{2}}\left[\ln x-\left(\ln x_{50,0}+3 \sigma^{2}\right)\right]^{2}\right]
$$

There are two principal ways in which this probability distribution function can be interpreted. In a number distribution, if we extracted one particle from a large volume of suspension the probability density function (PDF) expresses the probability that the selected particle has a radius between $x$ and $x+d x$, in which case 
$\frac{d n}{n}=q_{0}(x) x d \ln x$

In a volume distribution we have a PDF which expresses the probability that a small sample element of dispersed phase volume came from a particle with a radius in the range $x$ to $x+d x$. Here we have

$\frac{d \phi}{\phi}=q_{3}(x) x d \ln x$

For a given number distribution we can obtain the equivalent volume distribution (and vice versa) by combining eqs. (4, 9 and 10$)$,

$\frac{d \phi}{\phi}=\frac{x^{3}}{e^{3 \ln x_{50,3}+4.5 \sigma^{2}}} \frac{d n}{n}$

Fig. 1 shows two volume distributions with the same median $x_{50,3}$ value but different widths and Fig. 2 shows the volume distributions which result when number distributions of the same form as Fig. 1 are transformed to volume. The median $x_{50,3}$ shift and the constancy of the standard deviation are clear. Fig. 3 illustrates that the number-to-volume transformation obtained using eqs. (4 and 10) is in agreement with the result from eqs. (8 and 10).

\section{WAVE PROPAGATION MODELS}

There is a vast literature concerning ultrasonic wave propagation in colloidal mixtures, reviewed in [1]. The model most commonly used is due to Epstein and Carhart [12], and Allegra and Hawley [13], and is known as the ECAH model [7]. It is frequently extended by developing the expression for wavenumber using the formulation due to Lloyd and Berry [22], as outlined in [1]. The original ECAH model was limited to monodisperse mixtures but it can be extended (see [23]) to incorporate $J$ different size bins to get the complex wavenumber $\beta$. The same applies to the Lloyd and Berry result in which case the wavenumber becomes (see [23]):

$$
\frac{\beta^{2}}{k_{c}^{2}}=1+\sum_{j=1}^{J}\left[\begin{array}{l}
\frac{3 \phi_{j}}{i k_{c}^{3} x_{j}^{3}}\left(A_{0 j}+3 A_{1 j}+5 A_{2 j}\right) \\
-\frac{27 \phi_{j}^{2}}{k_{c}^{6} x_{j}^{6}}\left(A_{0 j} A_{1 j}+5 A_{1 j} A_{2 j}\right) \\
-\frac{54 \phi_{j}^{2}}{k_{c}^{6} x_{j}^{6}}\left(A_{1 j}^{2}+\frac{5}{3} A_{0 j} A_{1 j}+3 A_{1 j} A_{2 j}+\frac{115}{21} A_{2 j}^{2}\right)
\end{array}\right]
$$


where $\beta$ is the complex wavenumber in the mixture, $k_{c}$ is the compression wave number in the continuous phase, $\phi$ is the volume fraction of particles of radius $r, J$ is the number of bins, and $A_{0}, A_{1}$ and $A_{2}$ are the partial wave amplitude coefficients. The physical interpretation of these, and their calculation, are detailed in [23]. $A_{2}$ is the quadripole term which will be insignificant for the low ranges of $\left(k_{c} x\right)$ implicit in this paper. The complex wave number is

$\omega / c(\omega)+i \alpha(\omega)$

where $\omega$ is angular frequency, $c(\omega)$ is phase velocity, $\alpha(\omega)$ is the amplitude attenuation coefficient and $i=\sqrt{-1}$.

More recently a number of new works on acoustic wave propagation in particulate mixtures have appeared in the literature. Caleap et al [24] have derived effective wave number up to second order to describe the propagation of compressional waves in high concentration fluids. They have used a multiple scattering approach and have generalised it to include pair distribution functions that describe the correlation in spatial position of the spherical scatters. Luppé et al [25] have extended the scattering formulations for wave propagation such as ECAH. For solid-in liquid suspensions they allow the evanescent viscosity waves from a given particle to re-scatter and mode-convert back to compression waves when they impinge on neighbouring particles. In principle, either of these approaches could be employed for the computations in this study, although they will require some engineering development before they can be applied to particle sizing in an ultrasonic instrument. The original formulations of ECAH and Lloyd and Berry have been used with success for particle sizing for many years, both in the laboratory and in commercial instruments, so we have employed the latter for our computations, using eq. (12) with the data from Table I.

The multi-polar resonant scattering of emulsions in the Mie scattering regime $\left(k_{c} x>>1\right)$ have been investigated by Brunet et al [26] and Mascaro et al [27]. The works used Foldy's model [28] to derive the wavenumber of a particulate mixture and demonstrated, with excellent experimental verification, the resonance behaviour of relatively large particles, outside the size range of interest in this paper.

Eq. (12) implies a summation over $J$ size bins whereas model candidate PSDs are expressed as continuous functions. When a lognormal distribution in volume is used we have found that 
the modelled attenuation does not change significantly (by less than 1\%) if more than nine bins are employed. These are arranged in a simple logarithmic progression centred on the modal value $\ln x_{50,3}$ of the continuous PSD. The required bin centres are:

$$
\begin{aligned}
& \ln x=\ln x_{50.3}-3 \sigma, \frac{6 \sigma}{8}+\ln x_{50,3}-3 \sigma, \frac{12 \sigma}{8}+\ln x_{50,3}-3 \sigma, \frac{18 \sigma}{8}+\ln x_{50,3}-3 \sigma, \ln x_{50,3}, \\
& \frac{30 \sigma}{8}+\ln x_{50,3}-3 \sigma, \frac{36 \sigma}{8}+\ln x_{50,3}-3 \sigma, \frac{42 \sigma}{8}+\ln x_{50,3}-3 \sigma, \ln x_{50.3}+3 \sigma
\end{aligned}
$$

The bin sizes are equally spaced between $\ln x_{50,3}-3 \sigma$ and $\ln x_{50,3}+3 \sigma$ with bin width $6 \sigma / 8$. However, if the candidate distribution used in the modelling phase of the particle sizing operation were to be based on number then the number of size bins in the calculation would need to change, on the basis of the discussion in section II above. We know that we can model a volume based distribution successfully using

$$
\ln x_{50,3}-3 \sigma \leq \ln x \leq \ln x_{50,3}+3 \sigma
$$

Since the standard deviation does not alter between the two types of distribution it may be thought that the number of size bins need not change for the number distribution, at least in principle. However, the centre of the set of bins chosen for the number distribution should shift according to

$$
\ln x_{50,0}+3 \sigma^{2}-3 \sigma \leq \ln x \leq \ln x_{50,0}+3 \sigma^{2}+3 \sigma
$$

Table II shows the number of bins required for different values of $\sigma$ and for different ranges of $x$, given by $\ln x_{50,0} \pm 7 \sigma, \ln x_{50,0} \pm 8 \sigma$ and $\ln x_{50,0} \pm 10 \sigma$.The table was derived by first calculating the attenuation versus frequency graph up to $30 \mathrm{MHz}$ using 101 discrete size bins and then comparing this result with calculations using smaller numbers of bins, in the sequence $9,11,13 \ldots$ and so on. When the two calculations matched to within $1 \%$, the smaller number was entered into the table.

\section{PARTICLE SIZE DISTRIBUTION AND ATTENUATION}

We now investigate how the form of the PSD affects the attenuation spectrum. Ultrasound propagation in colloids is generally in the long wavelength regime $\left(k_{c} x<1\right)$ where simplified scattering theories of ultrasound propagation can be applied [31, 32]. This enables attenuation and phase velocity to be plotted against the scale parameter $x f^{1 / 2}$ which is proportional to both thermal and shear wavelengths in fluids [4]; it has the advantage that different combinations of particle size and frequency fall on the same curve [32]. Whilst we 
do plot data versus $x f^{1 / 2}$ as abscissa, all of the computations in this work were done using the full model of eq. (12).

The relationship between total attenuation for monodisperse silica against the scaling parameter $x f^{1 / 2}$ based on a lognormal distribution in volume and the physical data in Table I is shown in Fig.4. There are three distinct regions in the graphs see Kytömaa [33]: (i) The viscous region corresponds to large numbers of small particles where the viscous drag on the particles dominates over inertial forces and the shear wavenumber in the continuous phase is much greater than the particle radius $\left(\left|k_{s} x\right|<<1\right)$ [31]; (ii) A transition region in which the shear wavelength is commensurable with particle size $\left(\left|k_{s} x\right| \approx 1\right)$; and (iii) An inertial region corresponding to small numbers of large particles where inertia forces on the particles dominate over viscous drag and the shear wavelength is much greater than the particle radius $\left(\left|k_{s} x\right| \gg>1\right)$. The total attenuation is small for high and low values of $x f^{1 / 2}$ whilst it has a maximum value in the transition region.

Plots for three values of standard deviation $(0.25,0.5$, and 0.75$)$ are shown in Fig. 4. In the viscous region an increase in standard deviation causes a small but significant increase in attenuation due to the inclusion of more large particles in the PSD. Conversely, in the inertial region the attenuation falls slightly due to the incorporation of more small particles. These two effects oppose each other in the transition region, although the net effect is a decrease in attenuation at higher standard deviations. The corresponding plots for a lognormal distribution based on number are shown on Fig. 5. This shows a significant shift leftwards as standard deviation is increased, and this follows directly from section II. It could also be interpreted as the incorporation of larger numbers of smaller particles with increase in distribution width [34]. The differences between Figs. 4 and 5 have implications for particle sizing which we investigate next.

\section{CHOICE OF CANDIDATE PSD FORM}

The considerations above imply that either a number or a volume distribution could be used as candidate in any given particle sizing exercise. The result of the use of either would give parameters with different physical meanings, namely volume median or number median, and volume standard deviation or number standard deviation. It should be possible to transform from one result to the other using eqs(3-11). To illustrate this we have used the wave 
propagation model to simulate different scenarios in a particle sizing exercise; the simulations are in five stages:

(i) Choose an input PSD which represents that of the actual colloid - lognormal in volume or number. Discretise the distribution into appropriate size bins and calculate the 'measured' attenuation spectrum.

(ii) Choose a distribution type for the candidate PSD to be used at the modelling stage of the PSD estimation either matching the choice in (i), or not matching the original choice.

(iii) Adapt the candidate distribution to give the best match between modelled and 'measured' attenuation spectra.

(iv) If the 'measured' distribution is volume and the candidate is number, calculate the equivalent volume median $x_{50,3}$ from eq. (5). Conversely, if the 'measured' distribution is number and the candidate is volume, calculate the number median $x 50,0$ using eq. (6). These calculations are done in logarithmic size space, so if the candidate distribution was number then the equivalent volume median is

$$
x_{50,3}=\exp \left(\ln x_{50,0}+3 \sigma^{2}\right)
$$

and conversely for a volume candidate distribution the number median becomes

$$
x_{50,0}=\exp \left(\ln x_{50,3}-3 \sigma^{2}\right)
$$

The simulations have been done for an aqueous colloid of silica particles with a dispersed phase volume fraction of $3 \%$, a median radius of $200 \mathrm{~nm}$ and three values of standard deviation $(0.25,0.5$, and 0.75$)$. For each combination of PSD, four simulations were performed with either volume or number based distributions for the input and candidate PSDs; the results are shown in Tables III, IV, and V. On the tables the first two columns indicate the choices of input ('measured') and candidate PSDs. The third column gives the median radius that resulted from the particle sizing exercise, either $x_{50,3}$ or $x_{50,0}$ depending on the candidate distribution type. The fourth gives the median values calculated using eqs. (48). The fifth column gives the standard deviations that arose from the initial sizing procedure. The sixth column indicates the fitting error associated with the model adaptation part of the PSD estimation. This is a least square error and was calculated using the Marquardt algorithm $[9,10]$.

The tables indicate clearly that, whatever choice is made for the candidate PSD (volume or number), the median value for the other PSD type is easily obtainable from eqs. (5) and (7). They also show that the estimate of standard deviation is independent of the choice of 
candidate PSD, as expected. In all combinations of input and candidate PSDs, the fitting errors are very close in value for the two types of candidate.

\section{UNCERTAINTIES IN PHYSICAL PROPERTIES}

The ECAH model requires seven physical properties for each phase in a colloidal mixture [35]; they are: density, compressibility or compression modulus, viscosity or shear modulus, thermal conductivity, thermal volume expansion coefficient, specific heat, and the compression wave attenuation coefficient. The properties of many common materials are available in established reference works, for example [14, 15], whilst they may not be known for many modern materials and it may be difficult, expensive or even impossible to measure them. However, if the physics underpinning the scattering phenomena and hence the attenuation is dominated by one of the many phenomena implicit in ECAH, it may be possible to make reasonable estimates of the missing properties where these do not impinge significantly on the dominant physics. For instance, in emulsions thermal effects dominate through the partial wave coefficient $A_{0}$ and the density of the suspended particles is often close to that of the continuous phase [7, 35]. In solid in liquid suspensions the dominant phenomena are visco-inertial, through the coefficient $A_{1}$, and it would seem reasonable to assume that an accurate knowledge of the density of the particles and the viscosity of the continuous phase would be required and that the thermal properties would be less important. Hipp et al have developed these ideas and have used a perturbation technique to fit a group of unknown physical properties when the sizes of the suspended particles were known accurately [6]. Babick et al showed the degree of dependency of PSD on the material properties when attenuation is assumed to be caused by either thermal or viscoinertial losses [36]. In a particle sizing exercise on suspensions of organic crystals Mougin et al [37] found that the PSDs obtained were most sensitive to the densities of both phases of the suspension.

\section{A. Effect of parameter variation on attenuation}

To complement the earlier studies cited above we have conducted a sensitivity exercise for aqueous suspensions of silica particles. Three median radii were used $(0.01 \mu \mathrm{m}, 0.2 \mu \mathrm{m}$ and $0.6 \mu \mathrm{m})$; these were specifically chosen on the basis that they corresponded with the viscous, transition and inertial regions respectively. Computations were done for each of these for three standard deviations as before. For each distribution the attenuation was calculated up to $30 \mathrm{MHz}$ and the $10 \mathrm{MHz}$ component was selected for analysis. The computation was 
repeated for changes in the values of the physical properties of silica in the range 0 to $50 \%$ and for each of these the change in attenuation at $10 \mathrm{MHz}$ was expressed as a differential with respect to the change in the relevant physical property. The results are shown on Table VI for all of the physical properties except density and for a single PSD with median radius $0.01 \mu \mathrm{m}$ and a standard deviation 0.75 . The results for the other PSDs were similar and so are not included here. The principal conclusion from Table VI is that quite large variations in the physical properties have a minimal effect on the attenuation.

In order to investigate the sensitivity to density we have calculated the change in attenuation at $10 \mathrm{MHz}$ in an aqueous suspension of silica particles as the assumed density of the silica is changed by up to $\pm 20 \%$ about a 'true' value of $2185 \mathrm{kgm}^{-3}$. This has been done for the three scattering regions - viscous, transition and inertial, identified on Fig. 4. In each case four PSDs have been included - monodisperse, and three polydiserse with $\sigma$ equal to $0.25,0.5$ and 0.75, all assumed to be lognormal in volume. The results are shown on Figs. 6-8 from which it will be seen that the variation in attenuation is in all three cases commensurable with or greater than the change in density. The effect of distribution width is significant in the viscous region but negligible in the transition and inertial regions, as might be expected from the total attenuation curve shown on Fig. 4. These results imply that for solid in liquid suspensions an accurate value for the density of the dispersed phase is required. The effects on particle sizing of an erroneous choice for the density value are investigated in the next section.

\section{B. Effect of density variation on particle sizing}

We now investigate by simulation the effect that an erroneous setting of the density of silica would have on a PSD estimation. The PSDs input to the simulation were lognormal in volume. A group of median sizes was chosen to set the conditions at $10 \mathrm{MHz}$ to correspond to the viscous, transition and inertial regions, namely $0.01,0.2$ and $0.6 \mu \mathrm{m}$ respectively, as used in the previous calculation of attenuation change. For each median radius three values of standard deviation were used $(0.25,0.5$ and 0.75$)$, as before. The attenuation spectra were computed up to $30 \mathrm{MHz}$ and each was then subject to a particle sizing exercise with the candidate PSD lognormal in volume. Each PSD estimation was done for a range of density values which varied in $2 \%$ steps between 0 and $\pm 10 \%$ of the 'true' value used in the input PSD. The median and standard deviations were extracted from these estimated PSDs and expressed as a \% change with respect to the 'true' values initially input to the simulation to 
represent the colloid under test. The results for the median size are shown on Figs. 9-11 for the viscous, transition and inertial regions respectively. In all three regions the change in the estimated median size is commensurable with or greater than the change in the assumed value for density. In the viscous region (Fig. 9) the greatest change occurs for the wider PSD, when $\sigma=0.75$ whereas in the transition region the change is greatest for the narrowest distribution $(\sigma=0.25)$. This is likely to be due to the fact that, for a narrow distribution, the majority of particle sizes will actually be in the transition region. For a wider distribution there will be some spread of particle sizes about the transition, with smaller particles approaching the viscous regime and larger ones approaching the inertial regime. The change in median in the inertial region is similar for all three distribution widths. Further, it is interesting to note that the slopes of the curves for the viscous region are negative whereas those for the other two are positive. We believe that this phenomenon is related to the change in sign of the gradient between the viscous and inertial regions in Fig. 4

Equivalent results for the effect of density variation on the estimated standard deviation are shown on Figs. 12-14. In all three regions we note that the errors are high by comparison with the errors in choice of density value for the dispersed phase, and that the errors in the estimate are greatest for the narrowest distributions - that is with the lowest values for $\sigma$. The effects of density variation on the fitting error associated with the model adaptation part of the PSD estimation are shown in Figs.15-17. In the transition and inertial regions we note that the fitting errors are significantly increased with the errors in the density value. However, in the viscous region the fitting errors remain approximately equal with the density variation. This is more likely due to the effect of the viscous drag and inertial force on the three regions as discussed in section IV. These results confirm that a correct choice of density value is key to a successful particle sizing exercise.

\section{DISCUSSION}

This study has been concerned with the estimation of the particle size distributions (PSDs) in solid-in-liquid suspensions using ultrasonic attenuation as the basis. The initial focus was on the differences between the two types of candidate PSD - in volume or number, and a simple method to transform between the two. It emerged that the actual choice of PSD type did not matter, although it is to be noted that the parameters which result have different meanings between volume and number. Whatever the first choice of candidate PSD the parameters pertaining to the other type can be obtained by simple calculation, eqs. (5 and 7). 
It was shown on Fig. 4 that the expected attenuation is only a weak function of the distribution width. The small differences on the curves on Fig. 4 imply a requirement for very high accuracy and precision (bias and noise) in the measurement of the attenuation spectrum. Kalashnikov and Challis [11] have demonstrated that noise in the raw received ultrasonic data maps non-linearly into errors in the calculated attenuation spectrum, and that the errors so generated are minimised when the total ultrasonic path loss in the test medium is around 1 Np. It is often possible to meet this condition by adjustment of the acoustic path length between the transmitting and receiving transducers. In situations where this is not possible an alternative is to improve the overall system signal to noise ratio (SNR) by appropriate choice of signal processing schemes such as the use of binary codes and correlation techniques to obtain the raw ultrasonic signal, see Challis and Ivchenko [38].

The second part of the paper dealt with the frequently occurring problem of not knowing all of the physical properties of the solid phase of the test colloid. We have shown clearly that, in the case of a simple solid-in-liquid suspension, large changes in any of the physical properties except density do not have significant effects on calculated attenuation, and by implication would also not be significant in PSD estimation. It would therefore be possible to 'guestimate' the physical properties (excepting density) on the basis of known properties of chemically similar materials, and then to check the sensitivity to changes in these approximate values using the type of analysis presented here. However, the value for the density of the particles will be required to be known to an accuracy commensurable with that required for the estimates of the median size and the standard deviation of the PSD.

\section{CONCLUDING REMARKS}

This study was based on a single material - an aqueous suspension of silica particles. Whilst the detail of the results may be different for other materials, we suggest that the basic simulation methods we have presented with have broad application for solid-in-liquid suspensions. Similar considerations may apply to other types of mixture such as suspensions of soft solids or liquid droplets (emulsions). The results of such studies would be both interesting and useful, although different from the current case of solid particles.

\section{REFERENCES}


[1] R. E. Challis, M. J. W. Povey, M. L. Mather, and A. K. Holmes, "Ultrasound techniques for characterizing colloidal dispersions," Rep. Prog. Phys., vol. 68, no. 7, pp. 1541-1637, 2005.

[2] A. K. Holmes, R. E. Challis, and D. J. Wedlock, "A wide bandwidth study of ultrasound velocity and attenuation in suspensions: comparison of theory with experimental measurements," Journal of Colloid and Interface Science, vol. 156, no. 2, pp. 261-268, 1993.

[3] M. C. Davis, "Coal slurry diagnostics by ultrasound transmission," J. Acoust. Soc. Am., vol. 64, no. 2, pp. 406-410, 1978.

[4] D. J. McClements and M. J. W. Povey, "Scattering of ultrasound by emulsions," $J$. Phys. D: Appl. Phys, vol. 22, no. 1, pp. 38-47, 1989.

[5] A. K. Holmes, R. E. Challis, and D. J. Wedlock, "A wide-bandwidth ultrasonic study of suspensions: the variation of velocity and attenuation with particle size," Journal of Colloid and Interface Science, vol. 168, no. 2, pp. 339-348, 1994.

[6] A. K. Hipp, G. Storti, and M. Morbidelli, "Particle Sizing in Colloidal Dispersions by Ultrasound. Model Calibration and Sensitivity Analysis," J. Phys. D: Appl. Phys., vol. 15, no. 7, pp. 2338-2345, 1999.

[7] British-Standards-Institute, "Measurement and characterization of particles by acoustic methods-Part 1: Concepts and procedures in ultrasonic attenuation spectroscopy " BS ISO 20998-1 British Standards Institute, 2012.

[8] S. Meyer, S. Berrut, T. I. J. Goodenough, V. S. Rajendram, V. J. Pinfield, and M. J. W. Povey, "A comparative study of ultrasound and laser light diffraction techniques for particle size determination in dairy beverages," Meas. Sci. Technol., vol. 17, no. 2, pp. 289-297, 2006.

[9] D. W. Marquardt, "An algorithm for least-squares estimation of nonlinear parameters," Journal of the society for industrial and applied mathematics, vol. 11, no. 2, pp. 431-441, 1963.

[10] D. W. Marquardt, "Solution of nonlinear chemical engineering models," Chem. Eng. Prog., vol. 55, no. 6, pp. 65-70, 1959.

[11] A. N. Kalashnikov and R. E. Challis, "Errors and uncertainties in the measurement of ultrasonic wave attenuation and phase velocity," IEEE Trans. UFFC, vol. 52, no. 10, pp. 1754-1768, 2005.

[12] P. S. Epstein and R. R. Carhart, "The Absorption of Sound in Suspensions and Emulsions. I. Water Fog in Air," J. Acoust. Soc. Am, vol. 25, no. 3, pp. 553-565, 1953.

[13] J. R. Allegra and S. A. Hawley, "Attenuation of sound in suspensions and emulsions: theory and experiments " J.Acoust.Soc.Am., vol. 51, no. 5B, pp. 1545-1564, 1972.

[14] G. W. C. Kaye and T. H. Laby, Tables of physical and chemical constants, 16th ed. Harlow: Longman, 1995.

[15] D. R. Lide, CRC handbook of chemistry and physics : a ready-reference book of chemical and physical data, 91st ed. London CRC Press, 2010.

[16] F. Babick, M. Stintz, and A. Richter, "Ultrasonic Particle Sizing of Disperse Systems with Partly Unknown Properties," Part. Part. Syst. Charact., vol. 23, no. 2, pp. 175183, 2006.

[17] L. Silverman, C. E. Billings, and M. W. First, Particle size analysis in industrial hygiene. United states of America: Academic press, INC., 1971.

[18] E. Limpert, W. A. Stahel, and M. Abbt "Log-normal distributions across the sciences:keys and clues," Bioscience, vol. 51, no. 5, pp. 341-352, 2001. 
[19] L. B. Kiss, j. Söderlund, G. A. Niklasson, and C. G. Granqvist, "New approach to the origin of lognormal size distributions of nanoparticles," Nanotechnology, vol. 10, no. 1, pp. 25-28, 1999.

[20] British-Standards-Institute, "Representation of results of particle size analysis-Part 5: Methods of calculation relating to particle size analyses using logarithmic normal probability distribution," BS ISO 9276-5 British Standards Institute, 2005.

[21] K. Leschonski, " Representation and evaluation of particle size analysis data," Part. Charact., vol. 1, no. 1-4, pp. 89-95, 1984.

[22] P. Lloyd and M. V. Berry, "Wave propagation through an assembly of spheres Part IV. Relations between different scattering theories," Proc.Phys.Soc., vol. 91, no. 3, pp. 678-688, 1967.

[23] R. E. Challis, J. S. Tebbutt, and A. K. Holmes, " Equivalence between three scattering formulations for ultrasonic wave propagation in particulate mixtures," J. Phys., D: Appl. Phys., vol. 31, no. 24, pp. 3481-3497, 1998.

[24] M. Caleap, B. W. Drinkwater, and P. D. Wilcox, "Coherent acoustic wave propagation in media with pair-correlated spheres," J. Acoust. Soc. Am. , vol. 131, no. 3, pp. 2036-2047, 2012.

[25] F. Luppé, J. Conoir, and A. Norris, "Effective wave numbers for thermo-viscoelastic media containing random configurations of spherical scatterers," J Acoust Soc Am. , vol. 131, no. 2, pp. 1113-1120, 2012

[26] T. Brunet, S. Raffy, B. Mascaro, J. Leng, R. Wunenburger, O. Mondain-Monval, O. Poncelet, and C. Aristégui, "Sharp acoustic multipolar-resonances in highly monodisperse emulsions," Appl. Phys. Lett., vol. 101, no. 011913, pp. 1-4, 2012.

[27] B. Mascaro, T. Brunet, O. Poncelet, C. Aristégui, S. Raffy, O. Mondain-Monval, and J. Leng, "Impact of polydispersity on multipolar resonant scattering in emulsions," $J$. Acoust. Soc. Am. , vol. 133, no. 4, pp. 1996-2003, 2013.

[28] L. L. Foldy, "The Multiple Scattering of Waves. I. General Theory of Isotropic Scattering by Randomly Distributed Scatterers," Physical Review, vol. 67, no. 3-4, p. 107, 1945.

[29] V. A. Del Grosso and C. W. Mader, "Speed of sound in pure water," J. Acoust. Soc. Am., vol. 52, no. 5B, pp. 1442-1446, 1972.

[30] M. C. Smith and R. T. Beyer, "Ultrasonic absorption in water in the temperature range $0^{\circ}-80^{\circ}$ C," J. Acoust. Soc. Am., vol. 20, no. 5, pp. 608-610, 1948.

[31] M. J. W. Povey, Ultrasonic techniques for fluids characterization. San Diego, California, USA: Academic press, 1997.

[32] D. J. McClements, "Principles of ultrasonic droplet size determination in emulsions," Langmuir, vol. 12, no. 14, pp. 3458-3461, 1996.

[33] H. K. Kytömaa, "Theory of sound propagation in suspensions: a guide to particle size and concentration characterization," Powder technology, vol. 82, no. 1, pp. 115-121, 1995.

[34] R. Al-Lashi, "Novel Approaches to Ultrasonic Particle Sizing in Suspensions with Uncertain Properties, and to the Design of Ultrasonic Spectrometers," PhD Thesis, Electrical and Electronic Engineering Department, University of Nottingham, 2011.

[35] British-Standards-Institute, "Measurement and characterization of particles by acoustic methods-Part 2: Guidelines for linear theory," BS ISO 20998-2 British Standards Institute, 2012.

[36] F. Babick, F. Hinze, and S. Ripperger, "Dependence of ultrasonic attenuation on the material properties," Colloids and Surfaces A: Physicochemical and Engineering Aspects, vol. 172, no. 1-3, pp. 33-46, 2000. 
[37] P. Mougin, D. Wilkinson, K. J. Roberts, R. Jack, and P. Kippax, "Sensitivity of particle sizing by ultrasonic attenuation spectroscopy to material properties," Powder Technology, vol. 134, no. 3, pp. 243-248, 2003.

[38] R. E. Challis and V. G. Ivchenko, "Sub-threshold sampling in a correlation-based ultrasonic spectrometer," Meas. Sci. Technol., vol. 22, no. 2, pp. 1-12, 2011. 